\title{
Comprehensive Synthesis Study of Well-Dispersed and Solution-Processed Metal Nanowires for Transparent Heaters
}

\author{
Marco Bobinger (D), ${ }^{1}$ Vasileios Dergianlis $\left(\mathbb{D},{ }^{1}\right.$ Markus Becherer, ${ }^{1}$ and Paolo Lugli ${ }^{2}$ \\ ${ }^{1}$ Department of Electrical and Computer Engineering, Technical University of Munich, Theresienstr. 90, 80333 Munich, Germany \\ ${ }^{2}$ Faculty of Science and Technology, Free University of Bozen-Bolzano, Universitätsplatz 1, 39100 Bolzano, Italy \\ Correspondence should be addressed to Marco Bobinger; marco.bobinger@tum.de
}

Received 7 February 2018; Accepted 29 March 2018; Published 9 May 2018

Academic Editor: Andrew R. Barron

Copyright (C) 2018 Marco Bobinger et al. This is an open access article distributed under the Creative Commons Attribution License, which permits unrestricted use, distribution, and reproduction in any medium, provided the original work is properly cited.

\begin{abstract}
We review and extend the numerous polyol-based synthesis protocols and postdeposition treatments that have been reported for silver nanowires (AgNWs). After tailoring substantial process parameters such as the process time, temperature as well as the stirring speed, the polyvinylpyrrolidone (PVP) to silver nitrate $\left(\mathrm{AgNO}_{3}\right)$ ratio, and the type of PVP, a high mean aspect ratio of 800 could be achieved. Interestingly, the addition of a small portion of potassium chloride $(\mathrm{KCl})$ not only leads to a reduction in diameter but also extremely enhances the nanowire dispersion and its stability. This effect is attributed to the role of $\mathrm{KCl}$, which, along with PVP, acts as a cocapping agent. Furthermore, sprayed AgNW and copper nanowire (CuNW) films were tested as transparent heaters. The AgNWs could be operated at a power density of at least $0.72 \mathrm{~W} / \mathrm{cm}^{2}$, which corresponds to a temperature of $213^{\circ} \mathrm{C}$, whereas the CuNWs failed at a power density and temperature of $0.4 \mathrm{~W} / \mathrm{cm}^{2}$ and $150^{\circ} \mathrm{C}$, respectively. Interestingly, the degradation of AgNWs shows a step-wise behavior and is mainly dominated by fragmentation, while the CuNWs are gradually oxidized.
\end{abstract}

\section{Introduction}

Transparent conducting electrodes (TCEs) are commonly fabricated using materials such as carbon nanotubes (CNTs) [1], PEDOT:PSS [2], graphene [3], graphene oxide [4], indium tin oxide (ITO) [5], and metal nanowires (MNWs) such as AgNWs [6] and CuNWs [7], as well as hybrid systems that are composed of, for example, CuNWs/graphene oxide [8], AgNWs/PEDOT:PSS [9], and AgNWs/CNTs [10]. The applications for these materials range from transparent heaters [11, 12], transparent antennas [13, 14], thermoacoustic speakers [15, 16], solar cells [17], photodiodes [18], touch panels [19], microlenses [20], and OLEDs [1] to electromagnetic interference shielding [21] as well as piezo- [22] and pyroelectric [23] energy conversion.

Attributed to the electrooptical performance, flexibility as well as scalable synthesis protocols and deposition techniques, solution-based metal nanowires (MNWs) are one of the candidates to replace ITO, the prevailing TCE material [24]. In this work, a comprehensive study that reviews the substantial process parameters for the polyol-based synthesis and deposition of AgNWs is presented and compared with care to the extensive literature. As an important finding that extends the state-of-the-art for the AgNW synthesis, it was found that the addition of $\mathrm{KCl}$ leads to well-dispersed solutions that are stable for months. The role of $\mathrm{KCl}$ can be ascribed to the one of a cocapping agent that aids to direct the uniaxial wire growth. For a practical application, sprayed AgNW films were tested as transparent heaters and compared to CuNW-films. Transparent heaters have already been reported for applications such as defogging car or aircraft windows as well as advertisement boards and military optics like night vision devices and rifle scopes $[25,26]$. Interestingly, the films showed different degradation mechanism; that is, the failure of AgNW films is mainly induced by fragmentation, whereas the failure of CuNW-film is induced by a gradual oxidation.

This paper is organized as follows. Section 2 describes the experimental procedure for the synthesis, deposition, and the characterization of the MNW-films. In Section 3, the results 
for the synthesis of AgNWs are presented and compared with literature. In this section, $\mathrm{KCl}$ is ascribed the role of a cocapping agent, which is in accordance with the previously reported effect of adding potassium bromide $(\mathrm{KBr})$. The characterization of the TCE films is presented in Section 4 for different postdeposition treatments that enhance the electrooptical properties of the films. In Section 5, the AgNW films are tested as transparent heaters and compared with CuNW-based devices. Parameters that are relevant for the heater application such as the maximum power density, operation temperature, time-to-failure, and the degradation mechanism are discussed for the two materials.

\section{Experimental Section}

2.1. AgNW Synthesis and SEM-Imaging. For the materials, $\mathrm{AgNO}_{3}$ was used as the precursor and ethylene glycol (EG) as the solvent and reducing agent, PVP with a molecular weight of $360 \mathrm{k}$ was used as the capping agent, and copper(II) chloride $\left(\mathrm{CuCl}_{2}\right)$ was utilized as seeds. $\mathrm{KCl}$ was added to avoid the formation of an agglomerate during the synthesis. All materials were purchased from Sigma Aldrich and used without further purification. For the synthesis, $25 \mathrm{ml}$ EG with $0.135 \mathrm{mg} \mathrm{CuCl}{ }_{2}$ was heated in a Woulff bottle to a temperature of $175^{\circ} \mathrm{C}$ under stirring at $260 \mathrm{rpm}$ for $20 \mathrm{~min}$. Subsequently, $160 \mathrm{mg}$ of $\mathrm{AgNO}_{3}$ was dissolved in $10 \mathrm{ml}$ EG for $7 \mathrm{~min}$ in an ultrasonic bath. $126 \mathrm{mg}$ PVP was gradually added to $10 \mathrm{ml}$ EG under rigorous stirring. The PVP- and $\mathrm{AgNO}_{3}$-containing solutions were then separately injected into the Woulff bottle with two syringe pumps at a constant rate of $0.7 \mathrm{ml} / \mathrm{min}$. After the injection had been finished, the stirring was turned off and the wires were allowed to grow for $30 \mathrm{~min}$. Finally, the as-synthesized solution was drop-coated onto a highly doped silicon substrate and imaged with a Carl Zeiss Evo MA scanning electron microscope at an acceleration voltage of $7 \mathrm{kV}$.

2.2. AgNW Film-Fabrication and Posttreatments. In order to remove the insulating material EG and the capping agent PVP that surrounds the nanowires, $4 \mathrm{~g}$ of the as-synthesized solution was diluted in $10 \mathrm{~g}$ acetone and centrifuged for $5 \mathrm{~min}$ at $2000 \mathrm{rpm}$. The precipitate was diluted with $15 \mathrm{~g}$ deionized water and centrifuged for another $5 \mathrm{~min}$ at $6000 \mathrm{rpm}$. Finally, the precipitate was diluted with $5 \mathrm{~g}$ ethanol for the spraycoating. Transparent conducting films were spray-coated using a commercial handhold airbrush (Triplex II from Gabbert, Germany) with a nozzle orifice of $0.35 \mathrm{~mm}$. To enhance the drying of the films, the samples were placed on a hot plate at a temperature of $70^{\circ} \mathrm{C}$.

2.3. CuNW Synthesis and Film Fabrication. CuNWs were synthesized and spray-deposited to TCEs, in accordance with recently published work [27].

2.4. Electrooptical Characterization. The specular transmittance spectra were recorded from $350 \mathrm{~nm}$ to $800 \mathrm{~nm}$ using the light from a $300 \mathrm{~W}$ xenon arc lamp chopped at $210 \mathrm{~Hz}$, which passes through an Oriel Cornerstone $2601 / 4$ monochromator and illuminates a calibrated photodiode with a transconductance amplifier that is connected to an Oriel Merlin digital lock-in amplifier. The sheet resistances were measured using a four-point probe head from Jandel connected to a Keysight B2901A source measuring unit. A constant current of $100 \mu \mathrm{A}$ was sourced for all measurements.

2.5. Heater Characterization. For the use as heaters, the AgNW films on glass were contacted by copper tape and conductive silver ink, resulting in an actively heated area of $3.5 \times 5.0 \mathrm{~cm}^{2}$. The thickness of the glass substrate was $1.45 \mathrm{~mm}$. The heaters were driven by a Keithley 2602A Dualchannel SMU. Infrared (IR) images were recorded using a high resolution calibrated thermal imaging camera (Testo $890,640 \times 480$ pixels), which employs bolometric detectors. The temperatures were determined using a MATLAB script that processes the IR-images by averaging over an area of around $1.5 \times 1.5 \mathrm{~cm}^{2}$ in the center of the heater.

2.6. FTIR Measurements. Attenuated total reflection Fourier transform infrared spectra (ATR-FTIR) were recorded from $400-4000 \mathrm{~cm}^{-1}$ at a resolution of $2 \mathrm{~cm}^{-1}$ using a Bruker alpha equipped with a platinum ATR-module.

\section{Solution-Based Synthesis of Silver Nanowires}

As shown in Figure 1, AgNWs were synthesized with a process time of (a) $0 \mathrm{~min}$, (b) $10 \mathrm{~min}$, (c) $20 \mathrm{~min}$, and (d) $30 \mathrm{~min}$ at a process temperature of $167^{\circ} \mathrm{C}$. The process time refers to the duration after which the injection of PVP and $\mathrm{AgNO}_{3}$ was stopped. For a process time of $0 \mathrm{~min}$, only silver nanospheres are visible, whereas after $10 \mathrm{~min}$, rod shaped silver nanostructures form. After $20 \mathrm{~min}$, the synthesis can be considered to be completed for following reasons: (i) no difference with respect to the length of the wires can be recognized between a process time of $20 \mathrm{~min}$ and $30 \mathrm{~min}$ and (ii) compared to the portion of nanowires; there is only a negligible portion of nanosphere or nanorod shaped byproducts that could contribute to the wire growth. For all subsequent syntheses, the process time was kept at $30 \mathrm{~min}$ to ensure a completed wire growth. This time compares well or is even shorter than studies that report a duration of $30 \mathrm{~min}$ [28], $60 \mathrm{~min}[6,29,30]$, and $120 \mathrm{~min}$ [31] for their standard process.

After determining that a process time of $20-30 \mathrm{~min}$ is sufficient for a process temperature of $167^{\circ} \mathrm{C}$ to complete a synthesis, a temperature series was conducted in order to begin with the tailoring of the synthesis with regard to the wire aspect ratio. Figure 2 shows low and high magnification SEM images for the process temperatures (a) $151^{\circ} \mathrm{C}$, (b) $160^{\circ} \mathrm{C}$, (c) $165^{\circ} \mathrm{C}, 175^{\circ} \mathrm{C}$, and (e) $182^{\circ} \mathrm{C}$. The mean length, the mean diameter, and the corresponding aspect ratios are shown in Figure 2(f) (see supporting information Figure $\mathrm{S}-1$ for the length and diameter histograms). For a process temperature of $151^{\circ} \mathrm{C}$, no wires formed. This observation can be attributed either to a too low process temperature or to a too short process time. For a significantly longer process 


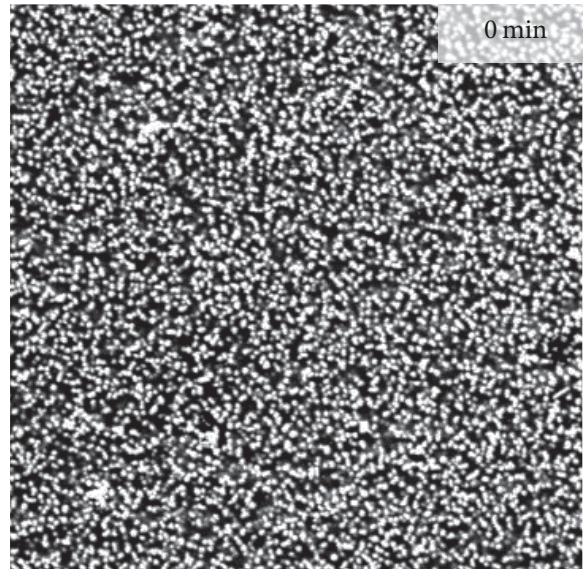

(a)

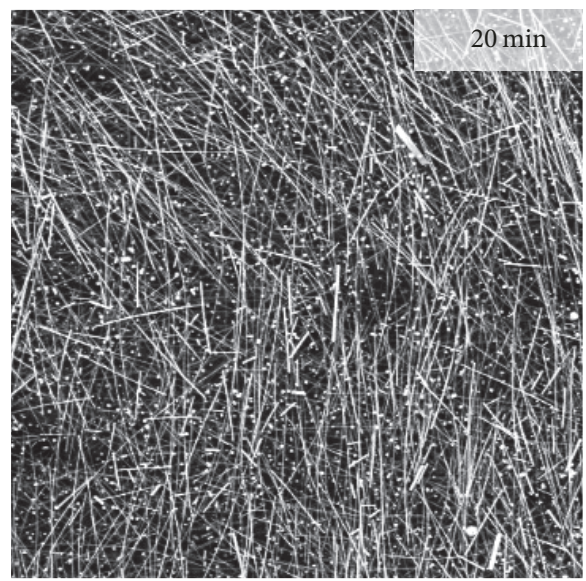

(c)

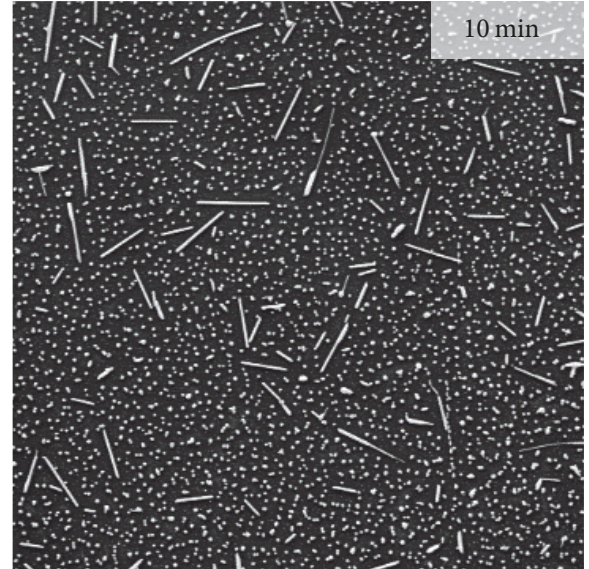

(b)

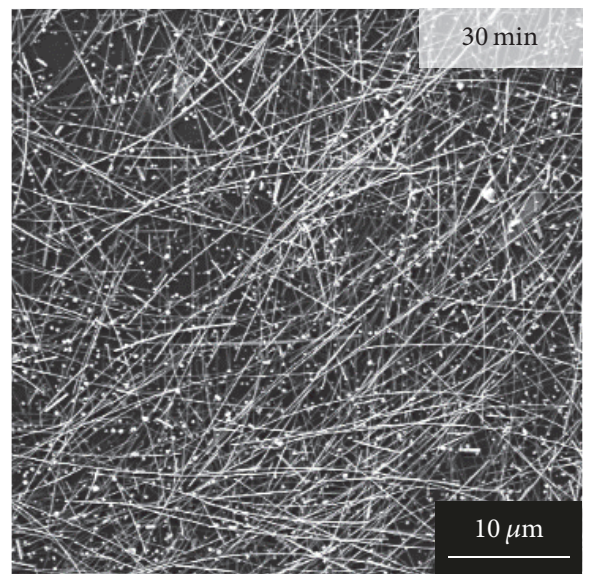

(d)

FIGURE 1: SEM images recorded after (a) $0 \mathrm{~min}$, (b) $10 \mathrm{~min}$, (c) $20 \mathrm{~min}$, and (d) $30 \mathrm{~min}$ after the injection of PVP and $\mathrm{AgNO}_{3}$ had been stopped. The process temperature was $167^{\circ} \mathrm{C}$. The scale bar in (d) applies to all images.

time and lower temperature of $12 \mathrm{~h}$ and $110^{\circ} \mathrm{C}$, respectively, we synthesized silver nanorods with a length of $6.0 \pm 2.8 \mu \mathrm{m}$ and a diameter of $0.43 \pm 0.17 \mu \mathrm{m}$, yielding to an aspect ratio of 14 (see supporting information Figure S-4 for SEM images and histograms). An optimum for the synthesis was identified around a growth temperature of $182^{\circ} \mathrm{C}$, which resulted in NWs with a length of $44.2 \pm 25 \mu \mathrm{m}$ and a diameter of $107 \pm 20 \mathrm{~nm}$ that correspond to an aspect ratio of 413 . The optimum growth temperature of $182^{\circ} \mathrm{C}$ compares well with the temperatures reported in literature that range from $152^{\circ} \mathrm{C}$ [32], $160^{\circ} \mathrm{C}[6,29]$, and $170^{\circ} \mathrm{C}[30]$ to $180^{\circ} \mathrm{C}[31]$. For this study, a careful temperature calibration was performed using a Pt100 thermoresistor that is placed in the heated EG solution to give the exact temperature. The occurrence of an optimum growth temperature was reported earlier $[28,33]$ and can be explained as follows: the higher the process temperature the higher the reduction of $\mathrm{Ag}^{+}-$ions to neutral $\mathrm{Ag}^{0}-$ atoms that can contribute to the wire formation faster and lead to longer wires. However, the binding of the capping agent to the (111) plane that should be reserved exclusively for $\mathrm{Ag}^{0}-$ atoms is also enhanced for increased temperatures [31]. The supply of
$\mathrm{Ag}^{0}$ - atoms that is enhanced for higher temperatures goes alongside with a decreased selectivity of the capping agent PVP, which in turn leads to an optimum for the temperature. Further, the effect of stirring during the synthesis is studied with regard to the wire diameter, length, and the generation of byproducts. Figure 3 shows the SEM images for (a) $10 \mathrm{~min}$ stirring during a total process time of $30 \mathrm{~min}$ at a stirring rate of $260 \mathrm{rpm}$ and stirring throughout the entire process time at a rate of (b) $260 \mathrm{rpm}$, (c) $500 \mathrm{rpm}$, and (d) $1000 \mathrm{rpm}$. When compared to the synthesis shown in Figure 2(e), which will be denoted as the reference synthesis in the following, the length of the wire decreases drastically from $44.2 \pm 25 \mu \mathrm{m}$ to $15.2 \pm 11 \mu \mathrm{m}$ even for the shortest stirring at the lowest speed (see supporting information Figure S-2 for the histograms). The reduction in length is accompanied by the generation of byproducts and an increase in the wire diameter, as shown in Figures 3(b) and 3(e). As depicted in Figure 3(c), at a stirring speed of $500 \mathrm{rpm}$, only rod shaped structures are visible, whereas for (d) at stirring speed of $1000 \mathrm{rpm}$, no wire or rod shaped structures can be recognized. The drastic reduction in length with increasing stirring speeds up to $1000 \mathrm{rpm}$ 


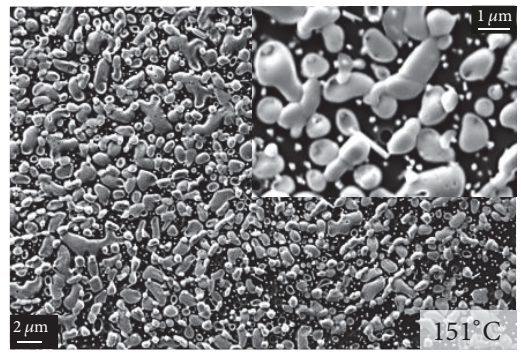

(a)

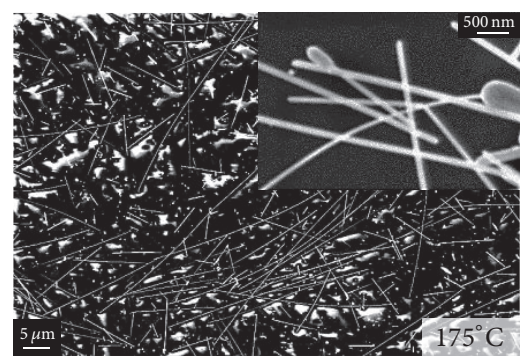

(d)

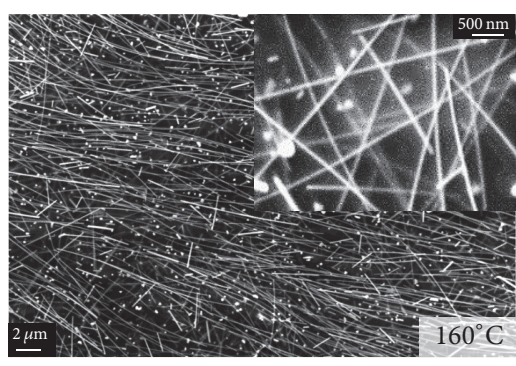

(b)

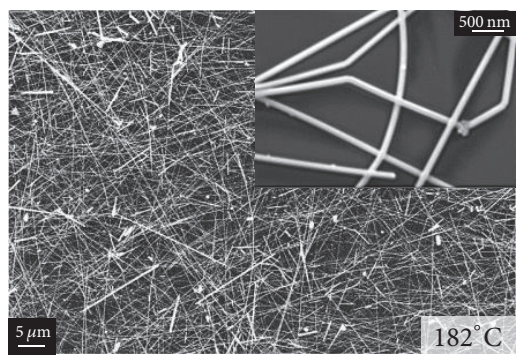

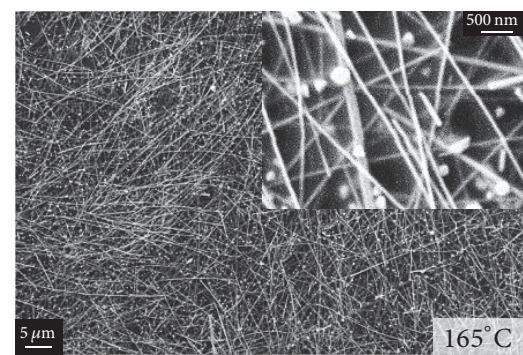

(c)

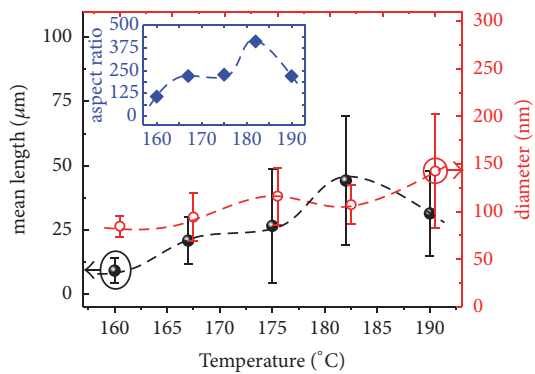

- Length

- diameter

- aspect ratio

(f)

Figure 2: SEM images recorded for process temperatures of (a) $151^{\circ} \mathrm{C}$, (b) $160^{\circ} \mathrm{C}$, (c) $165^{\circ} \mathrm{C}$, (d) $175^{\circ} \mathrm{C}$, and (e) $182^{\circ} \mathrm{C}$. (f) Mean length and mean diameter as a function of the process temperature. The inset shows the corresponding aspect ratio as a function of the process temperature.

has previously been reported and attributed to a mechanical interruption of the wire growth $[33,34]$. For the change in diameter with stirring speed, there are studies that report no dependency on the stirring speed [34] or even a reduction [33]. In our case, the aspect ratio is significantly lowered, as shown in the inset of Figure 3(e). On the one end, it can be concluded that stirring during the synthesis lowers the aspect ratio of the resulting nanowires and in turn their performance as a TCE with regard to a high transmittance and a low sheet resistance, as previously reported $[35,36]$. On the other hand, an increase in haze that is induced by increasing the diameters of the wires was reported to increase the light absorption of thin-film photovoltaic absorbers and thus increase their efficiency [37]. After the effect of stirring during the growth was studied, the influence of the precursor to capping agent weight ratio, that is, the $\mathrm{AgNO}_{3}$ to PVP weight ratio, was investigated. For the capping agent, we chose PVP with an average molecular (MW) weight of $360 \mathrm{kgmol}^{-1}$, referred to as PVP360k in the following, which is frequently reported in the literature for the polyol-based synthesis of AgNWs $[28,34,38-40]$. Besides, for PVP360k, numerous synthesis protocols have been proposed that employed PVPs with a different average MW that in turn corresponds to a different average chain length. The chain lengths of the PVP are composed of vinylpyrrolidone monomers with an elemental formula of $\mathrm{C}_{6} \mathrm{H}_{9} \mathrm{NO}$. Most studies report the use of PVP with a MW of $40 \mathrm{k}[6,30,31], 55 \mathrm{k}[29,32,35], 360 \mathrm{k}[38,40]$ as well as 1,3 M [41-43]. Song et al. [43] and Zhu et al. [44] investigated the effect of the MW of PVP on the yield and shape of AgNWs. In summary, they found that high yield and high aspect ratio AgNWs could be produced by changing the PVP MW from $40 \mathrm{k}$ and below to $200 \mathrm{k}, 800 \mathrm{k}$, or 1,3 M. These findings are in accordance with Figure S-5 that shows the SEM images for different MWs of $10 \mathrm{k}, 40 \mathrm{k}$ and $1,3 \mathrm{M}$. For $10 \mathrm{k}$ and $40 \mathrm{k}$, mostly sphere and rod shaped structures were observed, whereas for $1,3 \mathrm{M}$, the wires grew visibly longer. Figure 4 illustrates the SEM images for a series with a varying $\mathrm{AgNO}_{3}$ : PVP weight ratio of (a) $1: 3$, (b) $1: 6$, and (c) $1: 9$ along with the mean length and the mean diameter as a function of the $\mathrm{AgNO}_{3}$ : $\mathrm{PVP}$ weight ratio (see supporting information Figure S-3 for the histograms). The reference growth with a $\mathrm{AgNO}_{3}: \mathrm{PVP}$ ratio of $1: 1$ is depicted in Figure 2(e). The mean wire diameter was measured to be around $100 \mathrm{~nm}$, independent of the weight ratio, whereas the mean length showed an optimum for a weight ratio of $1: 6$. The optimum ratio resulted in wires with a mean length of $80 \pm 29 \mu \mathrm{m}$ and a mean diameter of $100 \pm 26 \mathrm{~nm}$, which yields to an average aspect ratio of 800 , that is, the ratio that was achieved for this work. Figure 4(e) schematically depicts the formation of a nanowire and aids to understand this effect: attributed to the lower surface atom density and in turn an increased reactivity of the (100)-plane $[29,39]$, the wire formation mostly occurs along the (111)-plane and PVP binds almost exclusively to the (100)-plane and passivates it $[6,35]$. Previous studies showed that PVP can interact with silver ions through polar groups such as the $>\mathrm{C}=\mathrm{O}$ carbonyl group [ 44 , 45] that are indicated by the red oxygen atoms in Figure 4(e). The more PVP molecules attach to the wire and the higher 


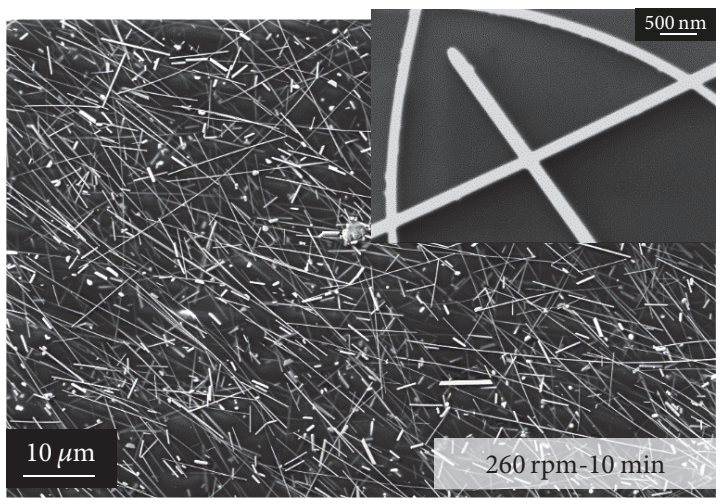

(a)

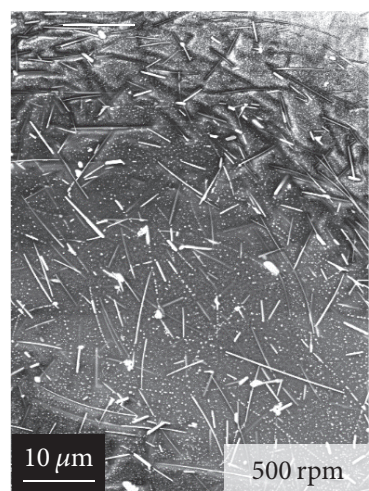

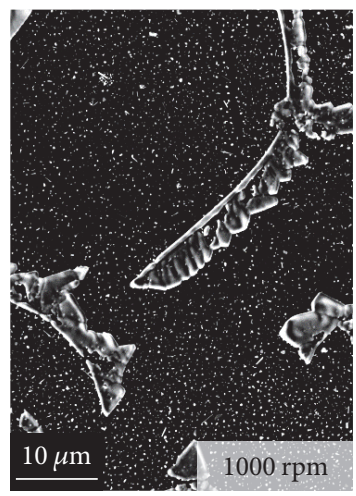

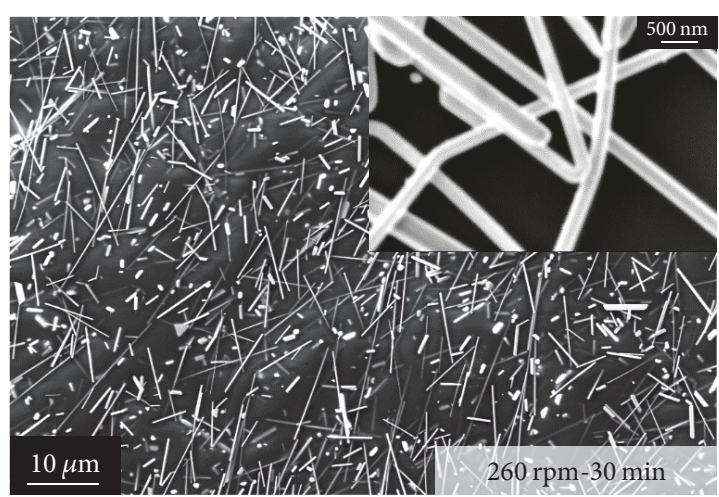

(b)

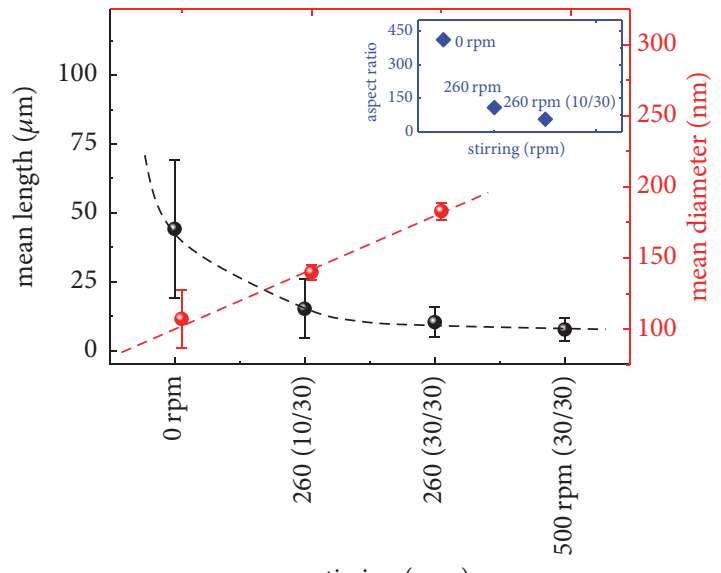

stirring (rpm)

- mean length

- mean diameter

- aspect ratio (c)

(d)

(e)

Figure 3: SEM images recorded for a stirring speed and stirring time of (a) $260 \mathrm{rpm}$ for $10 \mathrm{~min}$, (b) $260 \mathrm{rpm}$ for $30 \mathrm{~min}$, (c) $500 \mathrm{rpm}$ for $30 \mathrm{~min}$, and (d) $1000 \mathrm{rpm}$ for $30 \mathrm{~min}$ after the injection of PVP and $\mathrm{AgNO}_{3}$ has stopped. (e) Mean length and mean diameter as a function of the stirring speed. The inset shows the aspect ratio as a function of the stirring speed.

their degree of polymerization, the more carbonyl groups are present along the wire. These groups coordinate the supply of silver ions to the (100)-plane in a one-dimensional way [44] and aid to grow longer wires. However, if the concentration of PVP becomes too large, excess PVP also binds to the (111)-plane [33], which leads to a complete passivation of the wire and in turn ends the growth. Coskun et al. conducted a parameter study to determine the ideal molar ratio for $\mathrm{AgNO}_{3}:$ PVP in the range from $1: 3$ to $1: 11$ and found a molar ratio of $1: 7.5$, which corresponds to a weight ratio of $1: 5$, to be ideal with regard to the aspect ratio of the wires. This finding is in very good agreement with our optimum weight ratio of around $1: 6$ [33]. Finally, for the synthesis section of this work, the effect of the addition of the halide $\mathrm{KCl}$ was studied. So far, a few studies have presented the use of halides such as $\mathrm{KBr}$ for the synthesis of AgNWs and reported a reduction in diameter
$[28,31,42,46,47]$. For adding different concentrations of $\mathrm{KCl}$ (3-15 wt.\%, see supporting information Figure S-6), we have also observed a modulation in diameter, which lies in the range of 78-114 $\mathrm{nm}$. More interestingly, the addition of $\mathrm{KCl}$ greatly improved the nanowire dispersion and its stability (see the video in the supporting information that shows a $3 \mathrm{wt} . \%$ $\mathrm{KCl}$ growth over a duration of $30 \mathrm{~min}$ ). This effect becomes evident in Figure 5 that shows photos for an as-synthesized solution after two months for (a) $3 \mathrm{wt} . \%$ and (b) no addition of $\mathrm{KCl}$. Recently, Da Silva et al. [42] were able to describe the mechanism of introducing the halide $\mathrm{KBr}$ and synthesized wires with sub-20 nm diameters. In accordance with their findings and the schematic illustrated in Figure 5(c), the effect of adding $\mathrm{KCl}$ can be understood by ascribing two roles: (i) the formation of $\mathrm{AgCl}$ particles that continuously release $\mathrm{Ag}^{+}$-cations and in turn slow down the fast autocatalytic 


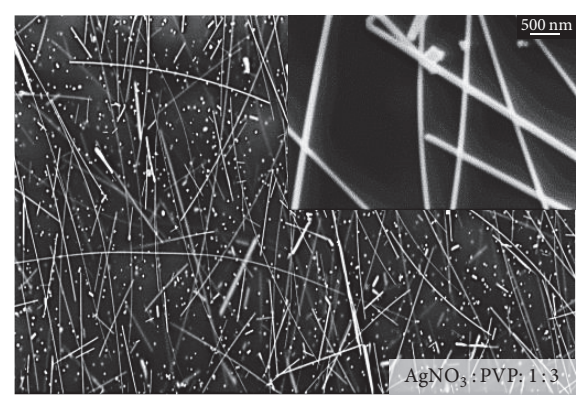

(a)

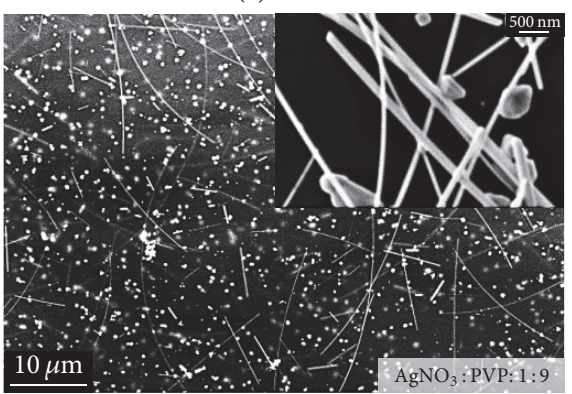

(c)

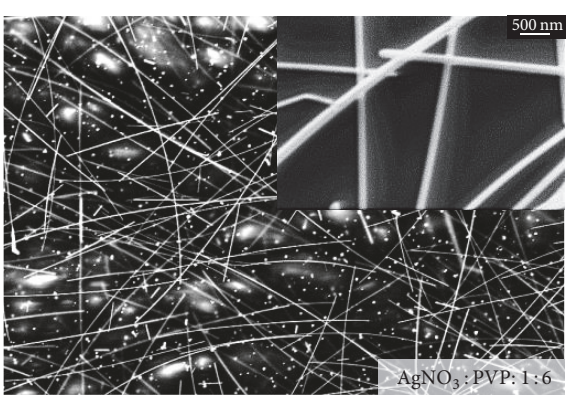

(b)

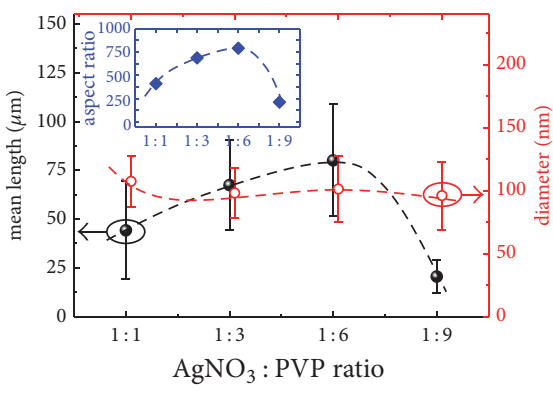

- mean length

- diameter

- aspect ratio

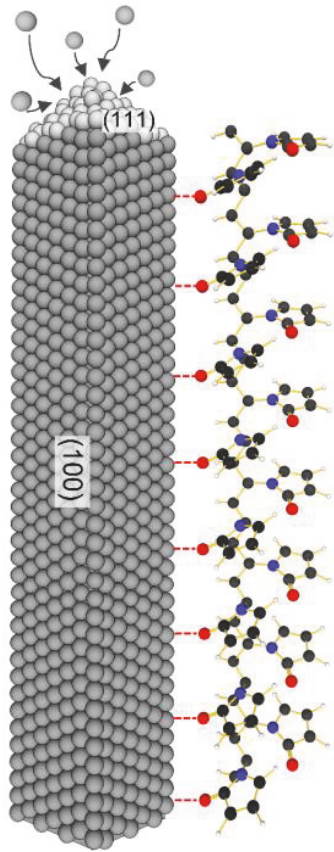

(d)

(e)

FIGURE 4: SEM images recorded for a $\mathrm{AgNO}_{3}$ : PVP weight ratio of (a) $1: 3$, (b) $1: 6$ and (c) $1: 9$. (d) Mean length and mean diameter as a function of the $\mathrm{AgNO}_{3}$ : PVP weight ratio. The inset shows the corresponding aspect ratio as a function of the $\mathrm{AgNO}_{3}: \mathrm{PVP}$ weight ratio. (e) Schematic drawing for the wire formation. (111) and (100) label the planes, where the wire formation and passivation occur. Grey spheres indicate silver atoms, whereas red, black, blue, and white spheres indicate oxygen, carbon, nitrogen, and hydrogen atoms from the PVP molecule, respectively. The scale bar in (c) also applies to (a) and (b).

reduction of $\mathrm{Ag}^{+}$-cations in the presence of EG [48] and (ii) the $\mathrm{Cl}^{-}$-anions represent a cocapping agent beside PVP that passivates the (100) plane. This observation is in accordance with previous studies that have already reported that ionic species such as halides can passivate the (100) plane and thus allow for anisotropic growth $[48,49]$. Since a good nanowire dispersion is a requirement for the later spraycoating process, $3 \mathrm{wt} . \% \mathrm{KCl}$ were used for the subsequent experiments.

\section{Fabrication of Transparent Conducting Films and Posttreatments}

In this section, an overview over various deposition techniques and treatments that are applied to enhance the electrooptical performance of AgNW based TCEs is presented. Further, the AgNW films that are fabricated in this study using a commercial handhold airbrush are characterized and the removal of PVP during an immersion treatment in water is studied in more detail. Solution-processed AgNWs were deposited to TCEs following several techniques such as (i) drop casting [50], (ii) Meyer rod coating [51], (iii) vacuum filtration [52], and (iv) spray-coating [53]. Attributed to a high homogeneity and the great upscaling potential, we applied a spray process utilizing a commercial handhold airbrush [54]. Due to the electrically insulating capping agent and solvent, that is, EG and PVP, the as-synthesized nanowires show a high resistance and cannot be used for most applications. Thus, a pre- or postdeposition treatment has to be applied [55]. As a predeposition treatment, the as-synthesized wires are usually centrifuged $1-3$ times in acetone and subsequently in either ethanol or water to remove EG and PVP $[6,33,42]$. As a postdeposition treatment, methods such as thermal sintering [56-58], photonic sintering [59-61], immersion in water [38], applying a conductive coating such as PEDOT:PSS [62-64], selective growth of silver nanoparticles at the AgNW junctions [65], and cold welding by applying a mechanical pressure $[38,52,66]$ have been reported.

The performance of a TCE is conventionally assessed by looking to the transmittance of the electrode at a wavelength of $550 \mathrm{~nm}$ and the sheet resistance. In accordance with the definition of a Figure of Merit (FoM) from Haacke, FoM = $T^{10} / R_{S}$ is defined [67]. FoMs for metal nanowires are usually reported in the range from 1000x FoM $=1-20$, whereas ITO shows values around 1000x FoM $=50$ [68]. Attributed to the continuous tailoring of the synthesis and posttreatment as well as purification protocols, FoMs as high as 1000x FoM $=30-34$ could be achieved $[37,69,70]$. The highest overall FoMs have been achieved for metal oxide/metal/metal oxide 


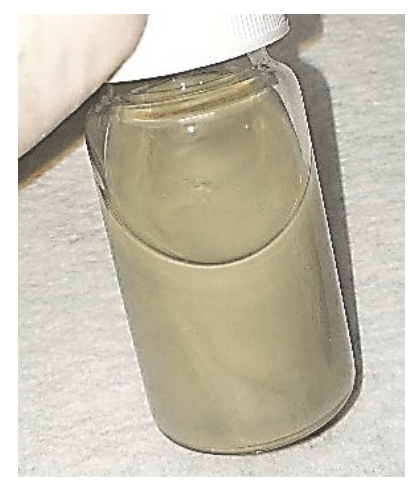

(a)

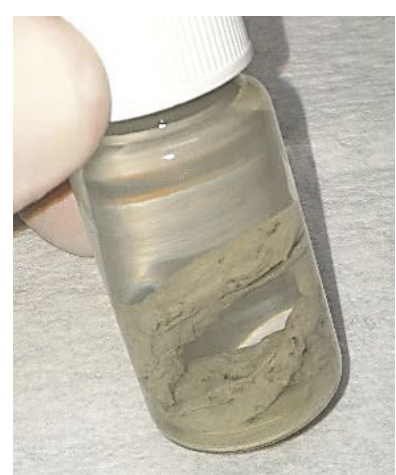

(b)

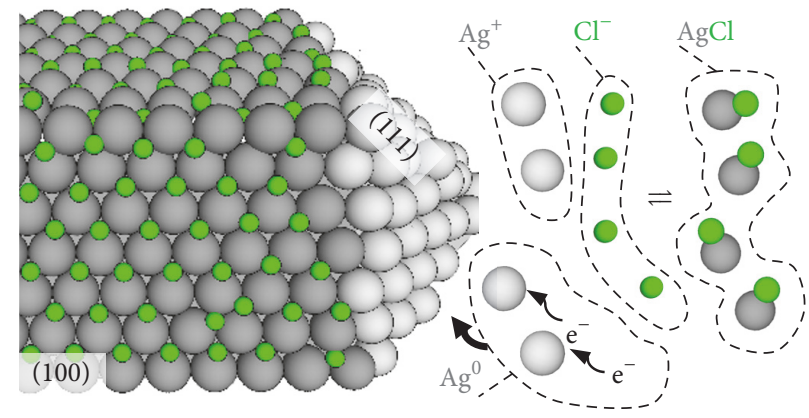

(c)

FIGURE 5: Photos of as-synthesized solutions after two months for the addition of $3 \mathrm{wt} \% \mathrm{KCl}$ and (b) without KCl. (c) Schematic for the effect of $\mathrm{KCl}$ on the wire formation, which can be grouped into two roles: (i) the binding of $\mathrm{KCl}$ on the (100) plane that results in the passivation of the plane and (ii) the formation of $\mathrm{AgCl}$ and release of $\mathrm{Ag}^{+}$-cations that are reduced to $\mathrm{Ag}^{0}$-atoms by EG. The latter effect limits the supply of $\mathrm{Ag}^{0}$-atoms and hence the growth of the NWs.

multilayer transparent electrodes with up to $1000 \mathrm{x}$ FoM $=78$ [71].

The specular transmittance spectra are shown in Figure 6(a) for films that were deposited with an increasing weight of the prepared spray ink. The spectra exhibit a transmittance valley around $320-400 \mathrm{~nm}$, which can be ascribed to the transverse and longitudinal modes of the AgNW plasmon resonance [72]. The broadening of this resonance is a characteristic for NW networks with a wide diameter distribution [28]. Beside the transmittance, another important parameter is the haze value, which is displayed in Figure 6(a) along with the transmittance data. The haze value gives a measure of the scattering of light by a transparent conductor (see supporting information Figure S-7 for a schematic of the measurement technique). This scattering leads to a reduction in sharpness and contrasts of objects viewed through the conductor [73].

The effect of commonly reported posttreatment procedures, that is, (i) immersion in water for a duration of $10 \mathrm{~min}$ followed by (ii) thermal annealing on a hot plate at a temperature of $150^{\circ} \mathrm{C}$ for $30 \mathrm{~min}$, is shown in Figure 6(b). The immersion treatment in water leads to an efficient dissolution of the insulating capping agent PVP and thus results in a decrease in resistance, in accordance with the literature. Subsequent annealing at $150^{\circ} \mathrm{C}$, the commonly reported glass transition temperature of PVP, aids to lower the resistance further by melting residual PVP and sintering the wire-to-wire junctions, which in turn increases the cross section of the wire-to-wire contact areas [55]. The effect of the water treatment is further studied by FTIR measurements, as shown in Figure 6(d) that depicts the spectra before and after immersion in water. Before immersion in water, a strong absorption band around a wavenumber of $1660 \mathrm{~cm}^{-1}$ and three weaker absorption bands around 1463, 1420, and $1290 \mathrm{~cm}^{-1}$ are visible in the FTIR spectra. In accordance with literature, the appearance of these bands confirms the presence of PVP. The band at $1660 \mathrm{~cm}^{-1}$ is attributed to the stretching vibration of the $\mathrm{C}=\mathrm{O}$ carbonyl group in $\mathrm{PVP}$, whereas the latter bands appear due to the vibration of the heterocyclic in PVP (see supporting information Figure S-8 for FTIR reference spectra recorded for different PVPs) [74]. After immersion in water, no absorption bands can be recognized, which proves the efficient removal of PVP. The AgNW film itself shows no fingerprints since metals are not active in the infrared region.

The transmittances and sheet resistances along with the haze values at a wavelength of $550 \mathrm{~nm}$ are depicted in Figure 6(c). Typical haze values for ITO lie below 3\%, which compares well to the TCE with the parameters $\left(R_{S} ; T\right.$; Haze $)=$ $(30 \Omega / \square ; 87 \% ; 2.6 \%)$. Lower resistances are in general traded off for lower transmittances and an increased haze value, for example, $\left(R_{S} ; T ;\right.$ Haze $)=(6.2 \Omega / \square ; 79 \% ; 5.5 \%)$, which yields to the highest FoM of 1000x FoM $=15.3$, which has been achieved for this study. 


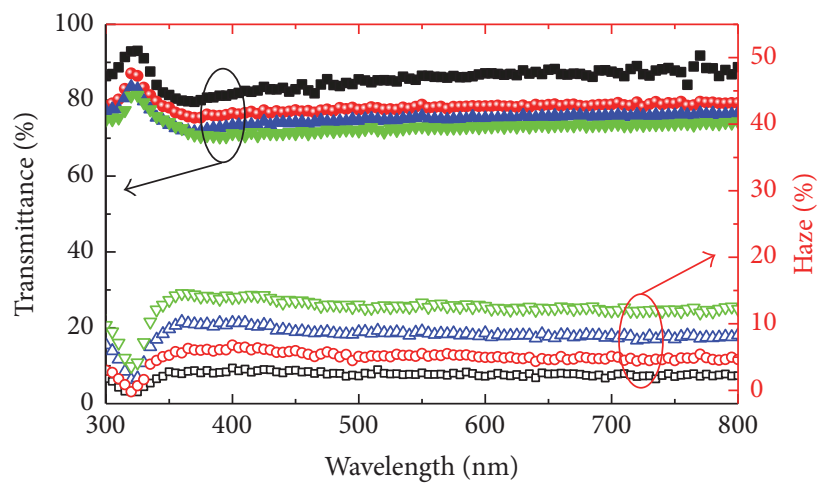

$\begin{array}{ll}T(\%) & \text { Haze }(\%) \\ -1 \mathrm{~g} & \circ 1 \mathrm{~g} \\ -2 \mathrm{~g} & \circ 2 \mathrm{~g} \\ \Delta 3 \mathrm{~g} & \triangle 3 \mathrm{~g} \\ \nabla 4 \mathrm{~g} & \nabla 4 \mathrm{~g}\end{array}$

(a)

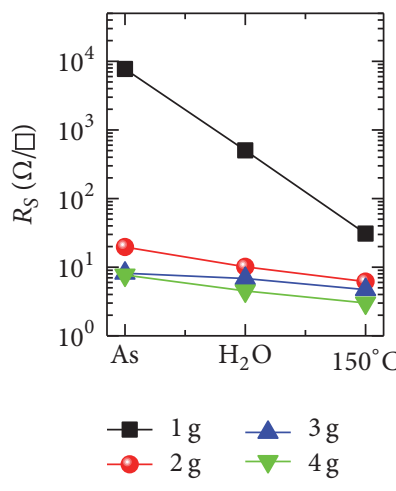

(b)

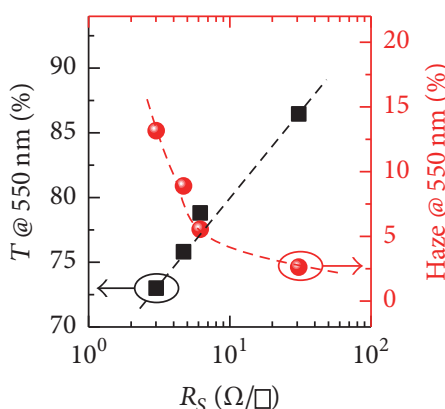

- $T @ 550 \mathrm{~nm}$

- Haze

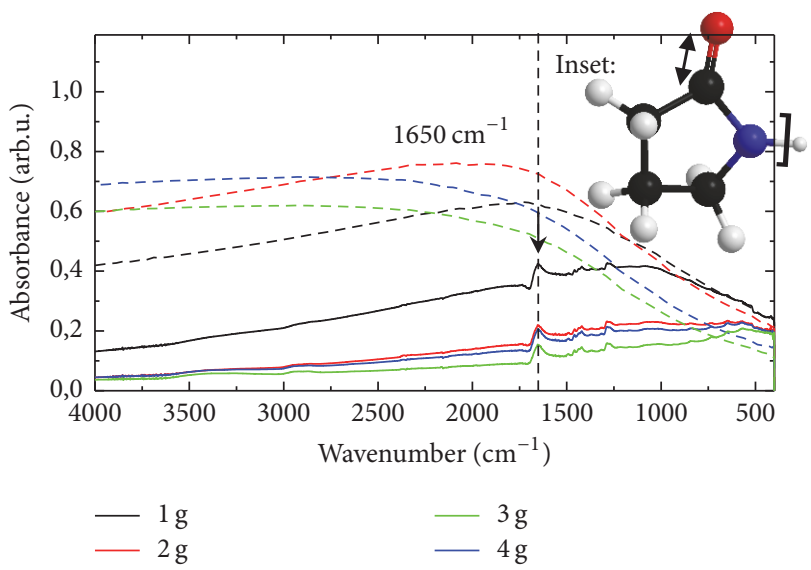

(d)

Figure 6: (a) Transmittance and haze spectra over the visible region for an increasing mass of sprayed AgNW ink. (b) $R_{S}$ for different postdeposition treatments (see main text for more details). (c) Transmittance and haze at a wavelength of $550 \mathrm{~nm}$ for different $R_{S}$. The dashed lines serve as a guide to the eye. (d) ATR-FTIR spectra for an increasing mass of sprayed AgNWs before (dashed lines) and after (solid lines) immersion in $\mathrm{H}_{2} \mathrm{O}$. The inset indicates the relevant part of the $\mathrm{PVP}$ repeating unit and the $\mathrm{C}=\mathrm{O}$ stretching mode that gives rise to the indicated absorbance peak around $1650 \mathrm{~cm}^{-1}$. Carbon, hydrogen, oxygen, and nitrogen atoms are colored black, white, red, and blue, respectively. 

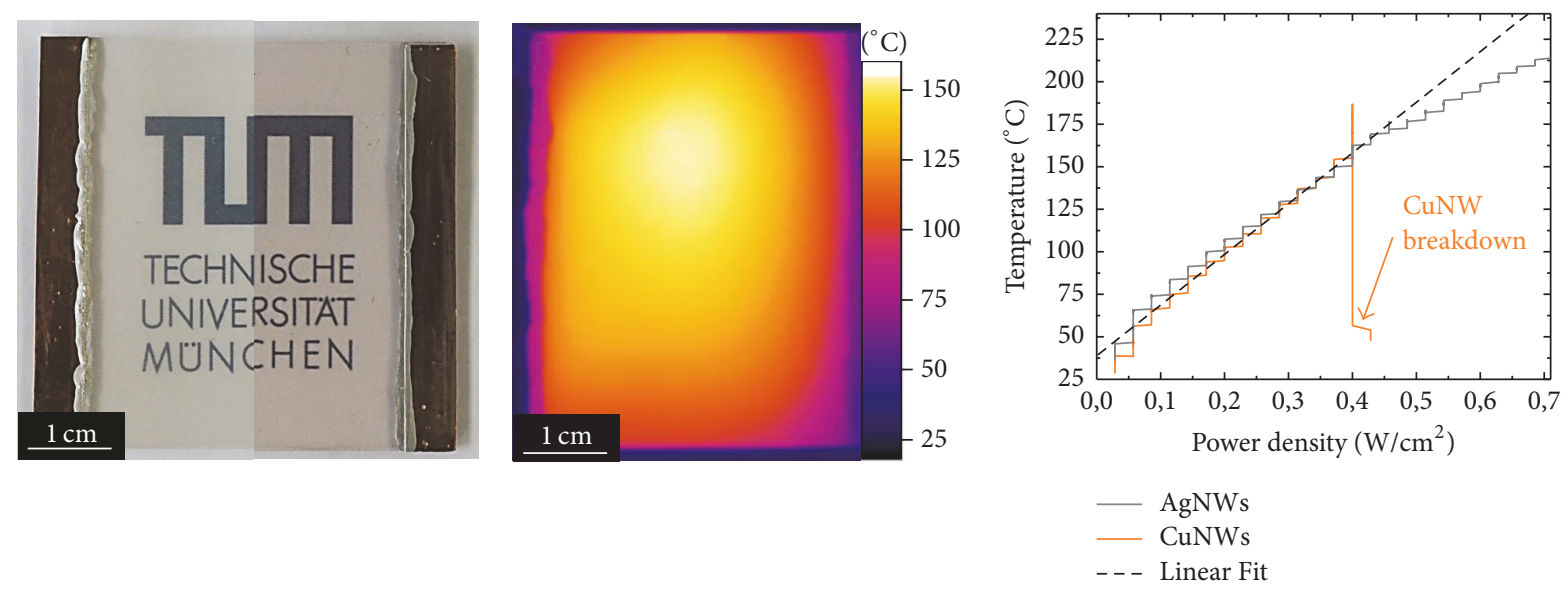

(a)

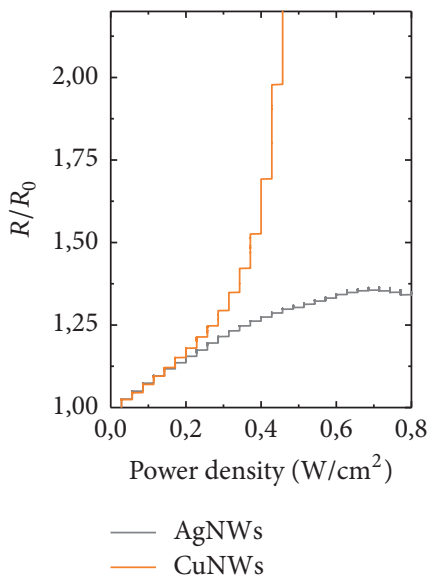

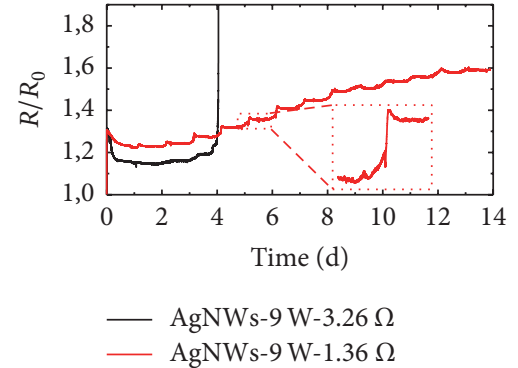

(e)

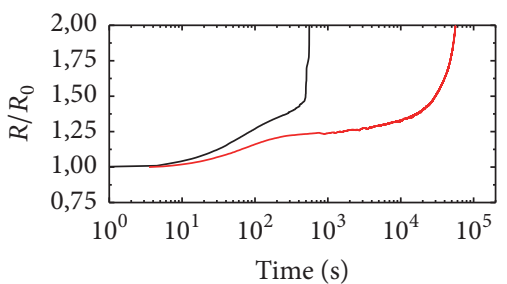

- CuNWs-9 W-4.2 $\Omega$

- CuNWs-6W-3.2 $\Omega$

(f) (c)

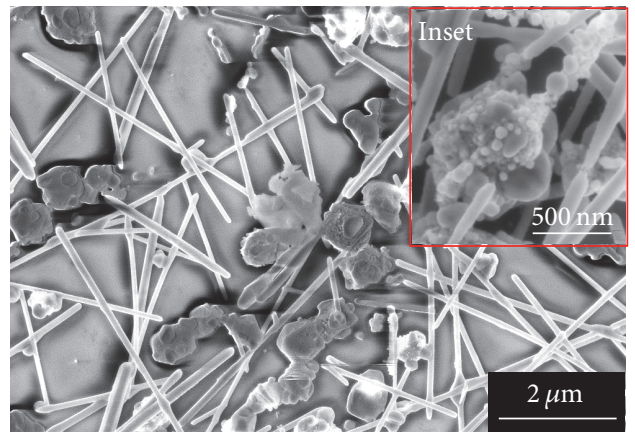

(g)

Figure 7: (a) On the left is a photo for a transparent heater based on AgNWs on glass substrate; on the right is a photo of a transparent heater based on CuNWs. (b) IR-image for an AgNW heater that is subjected to an electrical input power of $6 \mathrm{~W}$, which corresponds to a power density of $0.34 \mathrm{~W} / \mathrm{cm}^{2}$. (c) Temperature response as a function of the power density for an AgNW- and a CuNW-based heater. The dashed line represents a linear fit to the experimental data. (d) Increase in resistance, which was normalized with respect to the resistance at ambient conditions, for increasing power densities. Long-term degradation tests for AgNW- and CuNW-based heaters. (g) SEM images recorded for the $1.36 \Omega$ AgNW heater shown in (e). The inset shows a higher magnification SEM-image with silver sulfide nanoparticles.

\section{Transparent Heaters Based on MNWs}

Numerous studies have been presented for transparent heaters based on solution-processed AgNWs. So far, these studies focused on (i) fundamental research on AgNW based heaters deposited to rigid substrate such as glass [11, 26, 64], (ii) stretchability and bendability [75-77], (iii) textiles decorated with AgNWs, (iv) stability with regard to a long-term current induced chemical degradation and the maximum operating temperature, and (v) composite material systems such as AgNWs/PEDOT:PSS [64, 78], AgNWs/CNTs $[66,79]$, AgNWs/graphene [80], AgNW/clay platelets [11],
AgNWs/PMMA [81], and AgNWs/polyacrylate [77] that were reported to improve the adhesion, lower the resistance, and increase the chemical robustness of the devices. In this work, we present a comparison between AgNW- and CuNW-based heaters. The devices are tested with respect to their (i) heat distribution, (ii) long-term stability, and (iii) degradation mechanism that lead to device failures. Photos for (left) AgNW- and (right) CuNW-based heaters on glass substrate are illustrated in Figure 7(a). An IR-image recorded for an AgNW heater that is subjected to an electrical input power of $6 \mathrm{~W}$ is shown in Figure 7(b). Attributed to a homogeneous deposition process, that is, spray-coating, in 
combination with the homogeneous posttreatment process, that is, thermal annealing on a hot plate at a temperature of $150^{\circ} \mathrm{C}$, a homogeneous heat distribution could be achieved. The temperature profile is plotted in Figure 7 (c) as a function of the input power, which was normalized with respect to the actively heated area. The power was ramped in $500 \mathrm{~mW}$ steps in time spans of $30 \mathrm{~min}$. Both heaters show a similar temperature profile that can be approximated by a linear fit to the experimental data up to a power density of around $0.40 \mathrm{~W} / \mathrm{cm}^{2}$. The extracted slope for the temperature-powerdensity response yields to $298 \pm 3^{\circ} \mathrm{C} \mathrm{W} / \mathrm{cm}^{2}$, which is superior or compares well to the values reported for MNW- or metal-oxide-based devices that are typically reported in the range of $134-388^{\circ} \mathrm{CW} / \mathrm{cm}^{2}[64,82]$. At a power density of around $0.40 \mathrm{~W} / \mathrm{cm}^{2}$, which corresponds to a temperature of $150^{\circ} \mathrm{C}$, the CuNW-based heater turns nonconductive and fails. As studied for the case of thermal annealing of CuNWfilms under ambient air in an earlier work, this failure can be explained by a temperature-induced oxidation that becomes pronounced for temperatures above $150^{\circ} \mathrm{C}$ [27]. This degradation mechanism has also been reported by Zhai et al. for CuNW-films under current flows that correspond to temperatures above $80^{\circ} \mathrm{C}$ [83]. The failure of the CuNW-film goes along with a steep increase in resistance that is shown in Figure 7(d) for the AgNW- and the CuNW-based heaters. The AgNW heater can at least be operated up to a power density of $0.70 \mathrm{~W} / \mathrm{cm}^{2}$, which results in a temperature of $213^{\circ} \mathrm{C}$. Interestingly, the resistance curve of the AgNW heater in Figure 7 (d) shows a deviation from its linear behaviour towards a slower increase above a power density of $0.40 \mathrm{~W} / \mathrm{cm}^{2}$. This phenomenon is known as electrical sintering that lowers the contact resistances of the wire junctions and has already been reported for MNW-films [75, 84]. Moreover, the longterm stability of the films was studied, as shown in Figure 7 for (e) AgNWs and (f) CuNWs. Both AgNW films were subjected to an input power of $9 \mathrm{~W}$, which corresponds to a temperature of $183^{\circ} \mathrm{C}$. In accordance with previous studies, the film with the higher resistance and in turn a lower wire density shows an earlier failure, whereas the low-impedance film was operational even after 14 days. The step-wise increase in resistance is a strong indication that percolating paths are subsequently eliminated by fragmentation of the wires. As shown in (g), the wires are likely to melt at junctions. In nanowire networks, the junctions represent hot spots, since the heat of each neighbouring wire is transferred towards them. Beside the fragmentation, an effect that can add up to the increase in resistance is the chemical degradation of silver to silver sulfide $\left(\mathrm{Ag}_{2} \mathrm{~S}\right)$ due to reduced sulfur gases that are present in the ambient air, as previously reported. It is reported that this sulfurization goes along with the formation of $\mathrm{Ag}_{2} \mathrm{~S}$ that can be recognized in the inset in Figure 7(g) [85]. In contrast to the step-wise degradation of the AgNWs, the CuNWs first show a gradual increase in resistance followed by a fast breakdown, as shown in Figure 7(f). In accordance with literature and SEM images recorded after device failure (see supporting information Figure S-9), these results indicate that the CuNWs first undergo a long gradual oxidation followed by rapid fragmentation.

\section{Conclusion}

Our findings show that the addition of $\mathrm{KCl}$ leads to welldispersed and stable AgNW dispersions. This result can have an important impact to propel the commercialization of AgNWs as an ITO-replacement since shelf life and processability are important attributes that characterize the quality of MNW-based inks. In short- and long-term stress tests under high electrical power densities, the AgNWs proved to be much more resilient than CuNWs attributed to their chemical resistance.

\section{Data Availability}

Supporting information is available online or from the author.

\section{Conflicts of Interest}

The authors declare no conflicts of interest.

\section{Acknowledgments}

This work was supported by the German Research Foundation (DFG) and the Technical University of Munich within the funding programme Open Access Publishing.

\section{Supplementary Materials}

Figure S-1: histograms for (left) the length and (right) the diameter distributions for the temperature series shown in Figure 2. In accordance with Figure 2, the histograms are labeled with the corresponding temperature. Figure S-2: histograms for (left) the length and (right) the diameter distributions for the stirring series shown in Figure 3. In accordance with Figure 3, the histograms are labeled with the corresponding stirring parameters. Figure S-3: histograms for (left) the length and (right) the diameter distributions for the PVP: $\mathrm{AgNO}_{3}$ mass ratio series shown in Figure 4. In accordance with Figure 4, the histograms are labeled with the corresponding mass ratios. Figure S-4: histograms for (a) the length and (b) the diameter for a synthesis at a process temperature and time of $110^{\circ} \mathrm{C}$ and $12 \mathrm{~h}$, respectively. SEM images under (c) a low and (d) a high magnification for the low-temperature synthesis. Figure S-5: SEM images for syntheses that were conducted with a different average molecular weight for PVP of (a) $10 \mathrm{k}$, (b) $40 \mathrm{k}$, and (c) $1.3 \mathrm{M}$. For each synthesis, the weight content for $\mathrm{AgNO}_{3}$ and for PVP was kept constant. (d) mean length of the nanowires as a function of the average molecular weight. Figure S6: SEM images for syntheses that were conducted with a different weight ratio for $\mathrm{KCl}: \mathrm{AgNO}_{3}$ of (a) $3 \%$, (b) $9 \%$, and (c) $15 \%$. (d) Mean length and mean diameter as a function of the $\mathrm{KCl}: \mathrm{AgNO}_{3}$ weight ratio. The inset shows the corresponding aspect ratio as a function of the $\mathrm{KCl}: \mathrm{AgNO}_{3}$ weight ratio. Figure S-7: schematic for the four transmittance measurements, that is, $\left(T_{1}-T_{2}\right)$ that are required to determine the haze value. $(\mathrm{a}-\mathrm{d})$ indicate for which measurements the reflector and the sample are required. Figure S-8: ATR-FTIR measurements for different average molecular weights for 
PVP of $10 \mathrm{k}, 40 \mathrm{k}, 360 \mathrm{k}$ and $1.3 \mathrm{M}$. No substrate was utilized to avoid unwanted fingerprints. The PVP was pressed on the ATR-crystal in powder form. Figure S-9: SEM images recorded under (a) high and (b) low magnification for a CuNW-film heater that was subjected to an electrical input power of $6 \mathrm{~W}$ until device failure. (Supplementary Materials)

\section{References}

[1] J. Li, L. Hu, L. Wang, Y. Zhou, G. Grüner, and T. J. Marks, "Organic light-emitting diodes having carbon nanotube anodes," Nano Letters, vol. 6, no. 11, pp. 2472-2477, 2006.

[2] M. Vosgueritchian, D. J. Lipomi, and Z. Bao, "Highly conductive and transparent PEDOT:PSS films with a fluorosurfactant for stretchable and flexible transparent electrodes," Advanced Functional Materials, vol. 22, no. 2, pp. 421-428, 2012.

[3] X. Li, Y. Zhu, W. Cai et al., "Transfer of large-area graphene films for high-performance transparent conductive electrodes," Nano Letters, vol. 9, no. 12, pp. 4359-4363, 2009.

[4] Z. Yin, S. Sun, T. Salim et al., "Organic photovoltaic devices using highly flexible reduced graphene oxide films as transparent electrodes," ACS Nano, vol. 4, no. 9, pp. 5263-5268, 2010.

[5] L.-J. Meng and M. P. dos Santos, "Properties of indium tin oxide films prepared by rf reactive magnetron sputtering at different substrate temperature," Thin Solid Films, vol. 322, no. 1-2, pp. 56-62, 1998.

[6] Y. Sun, B. Gates, B. Mayers, and Y. Xia, "Crystalline silver nanowires by soft solution processing," Nano Letters, vol. 2, no. 2, pp. 165-168, 2002.

[7] A. R. Rathmell, S. M. Bergin, Y.-L. Hua, Z.-Y. Li, and B. J. Wiley, "The growth mechanism of copper nanowires and their properties in flexible, transparent conducting films," Advanced Materials, vol. 22, no. 32, pp. 3558-3563, 2010.

[8] L. Dou, F. Cui, Y. Yu et al., "Solution-processed copper/reducedgraphene-oxide core/shell nanowire transparent conductors," ACS Nano, vol. 10, no. 2, pp. 2600-2606, 2016.

[9] T. Tokuno, M. Nogi, J. Jiu, and K. Suganuma, "Hybrid transparent electrodes of silver nanowires and carbon nanotubes: a low-temperature solution process," Nanoscale Research Letters, vol. 7, p. 281, 2012.

[10] A. J. Stapleton, R. A. Afre, A. V. Ellis et al., "Highly conductive interwoven carbon nanotube and silver nanowire transparent electrodes," Science and Technology of Advanced Materials, vol. 14, no. 3, Article ID 035004, 2013.

[11] T. Kim, Y. W. Kim, H. S. Lee, H. Kim, W. S. Yang, and K. S. Suh, "Uniformly interconnected silver-nanowire networks for transparent film heaters," Advanced Functional Materials, vol. 23, no. 10, pp. 1250-1255, 2013.

[12] M. Bobinger, D. Angeli, S. Colasanti, P. La Torraca, L. Larcher, and P. Lugli, "Infrared, transient thermal, and electrical properties of silver nanowire thin films for transparent heaters and energy-efficient coatings," Physica Status Solidi (a), vol. 214, no. 1, Article ID 1600466, 2017.

[13] A. M. Olmos, J. A. Primicia, and J. L. F. Marron, "Simulation design of electrical capacitance tomography sensors," IET Science, Measurement \& Technology, vol. 1, no. 4, pp. 216-223, 2007.

[14] C. M. Lee, Y. Kim, Y. Kim, I. K. Kim, and C. W. Jung, "A flexible and transparent antenna on a polyamide substrate for laptop computers," Microwave and Optical Technology Letters, vol. 57, no. 5, pp. 1038-1042, 2015.
[15] L. Xiao, Z. Chen, C. Feng et al., "Flexible, stretchable, transparent carbon nanotube thin film loudspeakers," Nano Letters, vol. 8, no. 12, pp. 4539-4545, 2008.

[16] P. La Torraca, L. Larcher, M. Bobinger, P. Pavan, B. Seeber, and P. Lugli, "Physical modeling and characterization of thermoacoustic loudspeakers made of silver nano-wire films," Journal of Applied Physics, vol. 121, no. 21, Article ID 214502, 2017.

[17] Z. Yu, L. Li, Q. Zhang, W. Hu, and Q. Pei, "Silver nanowirepolymer composite electrodes for efficient polymer solar cells," Advanced Materials, vol. 23, no. 38, pp. 4453-4457, 2011.

[18] A. Falco, L. Cinà, G. Scarpa, P. Lugli, and A. Abdellah, "Fullysprayed and flexible organic photodiodes with transparent carbon nanotube electrodes," ACS Applied Materials \& Interfaces, vol. 6, no. 13, pp. 10593-10601, 2014.

[19] J. Lee, P. Lee, H. Lee, D. Lee, S. S. Lee, and S. H. Ko, "Very long Ag nanowire synthesis and its application in a highly transparent, conductive and flexible metal electrode touch panel," Nanoscale, vol. 4, no. 20, pp. 6408-6414, 2012.

[20] T. Krupenkin, S. Yang, and P. Mach, “Tunable liquid microlens," Applied Physics Letters, vol. 82, no. 3, pp. 316-318, 2003.

[21] T.-W. Lee, S.-E. Lee, and Y. G. Jeong, "Highly effective electromagnetic interference shielding materials based on silver nanowire/cellulose papers," ACS Applied Materials \& Interfaces, vol. 8, no. 20, pp. 13123-13132, 2016.

[22] T. Park, B. Kim, Y. Kim, and E. Kim, "Highly conductive PEDOT electrodes for harvesting dynamic energy through piezoelectric conversion," Journal of Materials Chemistry A, vol. 2, no. 15, pp. 5462-5469, 2014.

[23] T. Park, J. Na, B. Kim, Y. Kim, H. Shin, and E. Kim, "Photothermally activated pyroelectric polymer films for harvesting of solar heat with a hybrid energy cell structure," ACS Nano, vol. 9, no. 12, pp. 11830-11839, 2015.

[24] S. Ye, A. R. Rathmell, Z. Chen, I. E. Stewart, and B. J. Wiley, "Metal nanowire networks: The next generation of transparent conductors," Advanced Materials, vol. 26, no. 39, pp. 6670-6687, 2014.

[25] R. Gupta, K. D. M. Rao, S. Kiruthika, and G. U. Kulkarni, "Visibly transparent heaters," ACS Applied Materials \& Interfaces, vol. 8, no. 20, pp. 12559-12575, 2016.

[26] O. Ergun, S. Coskun, Y. Yusufoglu, and H. E. Unalan, "High-performance, bare silver nanowire network transparent heaters," Nanotechnology, vol. 27, no. 44, Article ID 445708, 2016.

[27] M. Bobinger, J. Mock, P. La Torraca, M. Becherer, P. Lugli, and L. Larcher, "Tailoring the aqueous synthesis and deposition of copper nanowires for transparent electrodes and heaters," Advanced Materials Interfaces, vol. 4, no. 20, Article ID 1700568, 2017.

[28] L. José Andrés, M. Fe Menéndez, D. Gómez et al., "Rapid synthesis of ultra-long silver nanowires for tailor-made transparent conductive electrodes: proof of concept in organic solar cells," Nanotechnology, vol. 26, no. 26, Article ID 265201, pp. 1-9, 2015.

[29] B. Wiley, Y. Sun, B. Mayers, and Y. Xia, "Shape-controlled synthesis of metal nanostructures: the case of silver," Chemistry, vol. 11, no. 2, pp. 454-463, 2005.

[30] Y. Gao, P. Jiang, L. Song et al., "Growth mechanism of silver nanowires synthesized by polyvinylpyrrolidone-assisted polyol reduction," Journal of Physics D: Applied Physics, vol. 38, no. 7, pp. 1061-1067, 2005.

[31] C. Chen, L. Wang, G. Jiang et al., "Study on the synthesis of silver nanowires with adjustable diameters through the polyol process," Nanotechnology, vol. 17, no. 15, pp. 3933-3938, 2006. 
[32] K. E. Korte, S. E. Skrabalak, and Y. Xia, "Rapid synthesis of silver nanowires through a $\mathrm{CuCl}$ - or $\mathrm{CuCl}_{2}$-mediated polyol process," Journal of Materials Chemistry, vol. 18, no. 4, pp. 437-441, 2008.

[33] S. Coskun, B. Aksoy, and H. E. Unalan, "Polyol synthesis of silver nanowires: An extensive parametric study," Crystal Growth and Design, vol. 11, no. 11, pp. 4963-4969, 2011.

[34] J. Jiu, T. Araki, J. Wang et al., "Facile synthesis of very-long silver nanowires for transparent electrodes," Journal of Materials Chemistry A, vol. 2, no. 18, pp. 6326-6330, 2014.

[35] J. H. Lee, P. Lee, D. Lee, S. S. Lee, and S. H. Ko, "Large-scale synthesis and characterization of very long silver nanowires via successive multistep growth," Crystal Growth \& Design, vol. 12, no. 11, pp. 5598-5605, 2012.

[36] H. Ding, Y. Zhang, G. Yang, S. Zhang, L. Yu, and P. Zhang, "Large scale preparation of silver nanowires with different diameters by a one-pot method and their application in transparent conducting films," RSC Advances, vol. 6, no. 10, pp. 80968102, 2016.

[37] C. Preston, Z. Fang, J. Murray et al., "Silver nanowire transparent conducting paper-based electrode with high optical haze," Journal of Materials Chemistry C, vol. 2, no. 7, pp. 1248-1254, 2014.

[38] T. Tokuno, M. Nogi, M. Karakawa et al., "Fabrication of silver nanowire transparent electrodes at room temperature," Nano Research, vol. 4, no. 12, pp. 1215-1222, 2011.

[39] J.-Y. Lin, Y.-L. Hsueh, J.-J. Huang, and J.-R. Wu, "Effect of silver nitrate concentration of silver nanowires synthesized using a polyol method and their application as transparent conductive films," Thin Solid Films, vol. 584, pp. 243-247, 2015.

[40] J. Jiu, K. Murai, D. Kim, K. Kim, and K. Suganuma, "Preparation of Ag nanorods with high yield by polyol process," Materials Chemistry and Physics, vol. 114, no. 1, pp. 333-338, 2009.

[41] Q. Huang, W. Shen, X. Fang et al., "Highly flexible and transparent film heaters based on polyimide films embedded with silver nanowires," RSC Advances, vol. 5, no. 57, pp. 4583645842, 2015.

[42] R. R. Da Silva, M. Yang, S.-I. Choi et al., "Facile synthesis of sub$20 \mathrm{~nm}$ silver nanowires through a bromide-mediated polyol method," ACS Nano, vol. 10, no. 8, pp. 7892-7900, 2016.

[43] Y.-J. Song, M. Wang, X.-Y. Zhang, J.-Y. Wu, and T. Zhang, "Investigation on the role of the molecular weight of polyvinyl pyrrolidone in the shape control of high-yield silver nanospheres and nanowires," Nanoscale Research Letters, vol. 9, no. 1, article 17, 8 pages, 2014.

[44] J. J. Zhu, C. X. Kan, J. G. Wan, M. Han, and G. H. Wang, "High-yield synthesis of uniform ag nanowires with high aspect ratios by introducing the long-chain PVP in an improved polyol process," Journal of Nanomaterials, vol. 2011, Article ID 982547, 7 pages, 2011.

[45] C. Kan, W. Cai, C. Li, and L. Zhang, "Optical studies of polyvinylpyrrolidone reduction effect on free and complex metal ions," Journal of Materials Research, vol. 20, no. 2, pp. 320$324,2005$.

[46] E.-J. Lee, Y.-H. Kim, D. K. Hwang, W. K. Choi, and J.-Y. Kim, "Synthesis and optoelectronic characteristics of $20 \mathrm{~nm}$ diameter silver nanowires for highly transparent electrode films," $R S C$ Advances, vol. 6, no. 14, pp. 11702-11710, 2016.

[47] K. Zhan, R. Su, S. Bai et al., "One-pot stirring-free synthesis of silver nanowires with tunable lengths and diameters: Via a Fe3+ \& Cl- co-mediated polyol method and their application as transparent conductive films," Nanoscale, vol. 8, no. 42, pp. 18121-18133, 2016.
[48] Q. Zhang, C. H. Moran, X. Xia, M. Rycenga, N. Li, and Y. Xia, "Synthesis of Ag nanobars in the presence of single-crystal seeds and a bromide compound, and their surface-enhanced raman scattering (SERS) properties," Langmuir, vol. 28, no. 24, pp. 9047-9054, 2012.

[49] Y. Xiong, H. Cai, B. J. Wiley, J. Wang, M. J. Kim, and Y. Xia, "Synthesis and mechanistic study of palladium nanobars and nanorods," Journal of the American Chemical Society, vol. 129, no. 12, pp. 3665-3675, 2007.

[50] J. Wang, J. Jiu, T. Sugahara et al., "Highly reliable silver nanowire transparent electrode employing selectively patterned barrier shaped by self-masked photolithography," ACS Applied Materials \& Interfaces, vol. 7, no. 41, pp. 23297-23304, 2015.

[51] C.-H. Liu and X. Yu, "Silver nanowire-based transparent, flexible, and conductive thin film," Nanoscale Research Letters, vol. 6, no. 1, article 75, 2011.

[52] T. Feng, Y. Wang, K. Wang, M. Qian, Y. Chen, and Z. Sun, "A facile method for preparing transparent, conductive, and paperlike silver nanowire films," Journal of Nanomaterials, vol. 2011, Article ID 935218, 5 pages, 2011.

[53] V. Scardaci, R. Coull, and J. N. Coleman, "Spray deposition of Silver Nanowire transparent conductive networks," in Proceedings of the 12th IEEE International Conference on Nanotechnology (NANO '12), pp. 2-4, August 2012.

[54] G. Y. Margulis, M. G. Christoforo, D. Lam et al., "Spray deposition of silver nanowire electrodes for semitransparent solidstate dye-sensitized solar cells," Advanced Energy Materials, vol. 3, no. 12, pp. 1657-1663, 2013.

[55] D. P. Langley, M. Lagrange, G. Giusti et al., "Metallic nanowire networks: Effects of thermal annealing on electrical resistance," Nanoscale, vol. 6, no. 22, pp. 13535-13543, 2014.

[56] P. N. Nirmalraj, A. T. Bellew, A. P. Bell et al., "Manipulating connectivity and electrical conductivity in metallic nanowire networks," Nano Letters, vol. 12, no. 11, pp. 5966-5971, 2012.

[57] T.-B. Song, Y. Chen, C.-H. Chung et al., "Nanoscale joule heating and electromigration enhanced ripening of silver nanowire contacts," ACS Nano, vol. 8, no. 3, pp. 2804-2811, 2014.

[58] B. Bari, J. Lee, T. Jang et al., "Simple hydrothermal synthesis of very-long and thin silver nanowires and their application in high quality transparent electrodes," Journal of Materials Chemistry A, vol. 4, no. 29, pp. 11365-11371, 2016.

[59] J. Jiu, T. Sugahara, M. Nogi et al., "High-intensity pulse light sintering of silver nanowire transparent films on polymer substrates: The effect of the thermal properties of substrates on the performance of silver films," Nanoscale, vol. 5, no. 23, pp. 11820-11828, 2013.

[60] J. A. Spechler, K. A. Nagamatsu, J. C. Sturm, and C. B. Arnold, "Improved efficiency of hybrid organic photovoltaics by pulsed laser sintering of silver nanowire network transparent electrode," ACS Applied Materials \& Interfaces, vol. 7, no. 19, pp. 10556-10562, 2015.

[61] J. Jiu, M. Nogi, T. Sugahara et al., "Strongly adhesive and flexible transparent silver nanowire conductive films fabricated with a high-intensity pulsed light technique," Journal of Materials Chemistry, vol. 22, no. 44, pp. 23561-23567, 2012.

[62] D. Y. Choi, H. W. Kang, H. J. Sung, and S. S. Kim, "Annealingfree, flexible silver nanowire-polymer composite electrodes via a continuous two-step spray-coating method," Nanoscale, vol. 5, no. 3, pp. 977-983, 2013.

[63] Y.-S. Liu, J. Feng, X.-L. Ou, H.-F. Cui, M. Xu, and H.B. Sun, "Ultrasmooth, highly conductive and transparent 
PEDOT:PSS/silver nanowire composite electrode for flexible organic light-emitting devices," Organic Electronics: Physics, Materials, Applications, vol. 31, pp. 247-252, 2016.

[64] S. Ji, W. He, K. Wang, Y. Ran, and C. Ye, “Thermal response of transparent silver nanowire/PEDOT:PSS film heaters," Small, vol. 10, no. 23, pp. 4951-4960, 2014.

[65] H. Lu, D. Zhang, X. Ren, J. Liu, and W. C. H. Choy, "Selective growth and integration of silver nanoparticles on silver nanowires at room conditions for transparent nano-network electrode," ACS Nano, vol. 8, no. 10, pp. 10980-10987, 2014.

[66] M.-X. Jing, C. Han, M. Li, and X.-Q. Shen, "High performance of carbon nanotubes/silver nanowires-PET hybrid flexible transparent conductive films via facile pressing-transfer technique," Nanoscale Research Letters, vol. 9, no. 1, p. 588, 2014.

[67] G. Haacke, "New figure of merit for transparent conductors," Journal of Applied Physics, vol. 47, no. 9, pp. 4086-4089, 1976.

[68] D. Langley, G. Giusti, C. Mayousse, C. Celle, D. Bellet, and J.-P. Simonato, "Flexible transparent conductive materials based on silver nanowire networks: A review," Nanotechnology, vol. 24, no. 45, Article ID 452001, 2013.

[69] B. Li, S. Ye, I. E. Stewart, S. Alvarez, and B. J. Wiley, "Synthesis and purification of silver nanowires to make conducting films with a transmittance of 99\%," Nano Letters, vol. 15, no. 10, pp. 6722-6726, 2015.

[70] S. B. Sepulveda-Mora and S. G. Cloutier, "Figures of merit for high-performance transparent electrodes using dip-coated silver nanowire networks," Journal of Nanomaterials, vol. 2012, Article ID 286104, 7 pages, 2012.

[71] S. Yu, L. Li, X. Lyu, and W. Zhang, "Preparation and investigation of nano-thick FTO/Ag/FTO multilayer transparent electrodes with high figure of merit," Scientific Reports, vol. 6, no. 1, Article ID 20399, 2016.

[72] Q. N. Luu, J. M. Doorn, M. T. Berry, C. Jiang, C. Lin, and P. S. May, "Preparation and optical properties of silver nanowires and silver-nanowire thin films," Journal of Colloid and Interface Science, vol. 356, no. 1, pp. 151-158, 2011.

[73] F. Cui, Y. Yu, L. Dou et al., "Synthesis of ultrathin copper nanowires using tris(trimethylsilyl)silane for high-performance and low-haze transparent conductors," Nano Letters, vol. 15, no. 11, pp. 7610-7615, 2015.

[74] R. Seoudi, A. A. Fouda, and D. A. Elmenshawy, "Synthesis, characterization and vibrational spectroscopic studies of different particle size of gold nanoparticle capped with polyvinylpyrrolidone," Physica B: Condensed Matter, vol. 405, no. 3, pp. 906-911, 2010.

[75] C. Celle, C. Mayousse, E. Moreau, H. Basti, A. Carella, and J.P. Simonato, "Highly flexible transparent film heaters based on random networks of silver nanowires," Nano Research, vol. 5, no. 6, pp. 427-433, 2012.

[76] S. Wang, X. Zhang, and W. Zhao, "Flexible, transparent, and conductive film based on random networks of Ag nanowires," Journal of Nanomaterials, vol. 2013, Article ID 456098, 6 pages, 2013.

[77] J. Li, J. Liang, X. Jian, W. Hu, J. Li, and Q. Pei, “A flexible and transparent thin film heater based on a silver nanowire/heatresistant polymer composite," Macromolecular Materials and Engineering, vol. 299, no. 11, pp. 1403-1409, 2014.

[78] X. He, R. He, Q. Lan et al., "Screen-printed fabrication of PEDOT: PSS/silver nanowire composite films for transparent heaters," Materials, vol. 10, no. 3, article 220, 2017.
[79] D. Kim, L. Zhu, D. Jeong et al., "Transparent flexible heater based on hybrid of carbon nanotubes and silver nanowires," Carbon, vol. 63, pp. 530-536, 2013.

[80] X. Zhang, X. Yan, J. Chen, and J. Zhao, "Large-size graphene microsheets as a protective layer for transparent conductive silver nanowire film heaters," Carbon, vol. 69, no. 2, pp. 437-443, 2014.

[81] X. He, A. Liu, X. Hu et al., "Temperature-controlled transparent-film heater based on silver nanowire-PMMA composite film," Nanotechnology, vol. 27, no. 47, Article ID 475709, 2016.

[82] I. S. Gainutdinov, E. A. Nesmelov, R. D. Aliakberov, and A. V. Mikhailov, "Heater elements based on In2O3(Sn) layers transparent in the visible region," Journal of Optical Technology, vol. 71, no. 10, p. 705, 2004.

[83] H. Zhai, R. Wang, X. Wang, Y. Cheng, L. Shi, and J. Sun, "Transparent heaters based on highly stable Cu nanowire films," Nano Research, vol. 9, no. 12, pp. 3924-3936, 2016.

[84] T. Sannicolo, D. Munoz-Rojas, N. D. Nguyen et al., "Direct imaging of the onset of electrical conduction in silver nanowire networks by infrared thermography: evidence of geometrical quantized percolation," Nano Letters, vol. 16, no. 11, pp. 70467053, 2016 .

[85] J. L. Elechiguerra, L. Larios-Lopez, C. Liu, D. Garcia-Gutierrez, A. Camacho-Bragado, and M. J. Yacaman, "Corrosion at the nanoscale: The case of silver nanowires and nanoparticles," Chemistry of Materials, vol. 17, no. 24, pp. 6042-6052, 2005. 


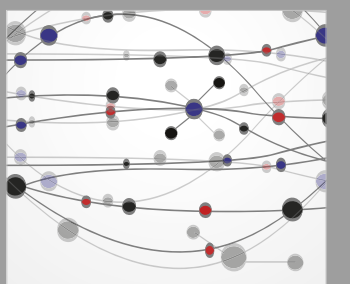

The Scientific World Journal
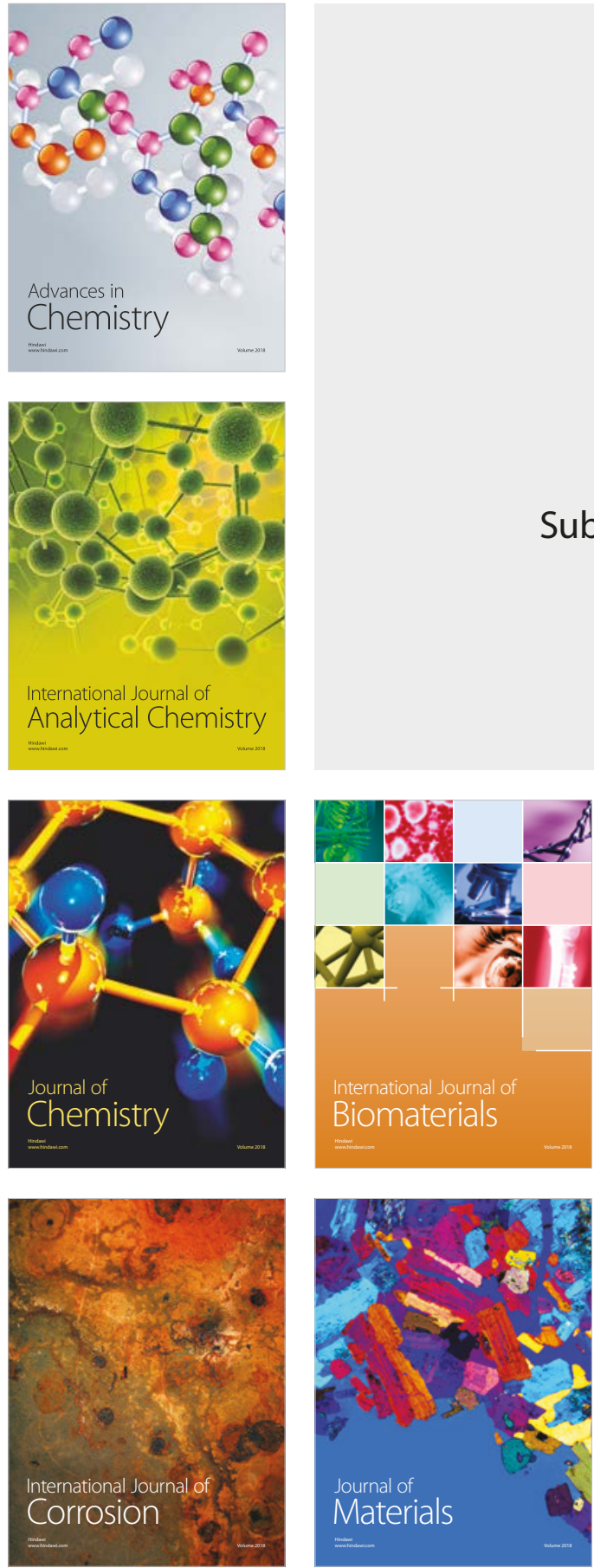

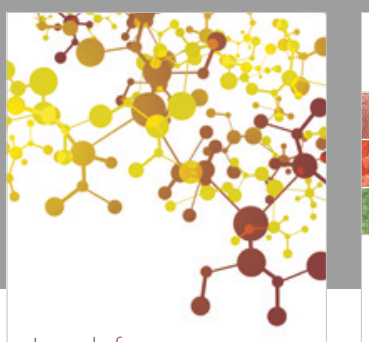

Journal of

Applied Chemistry
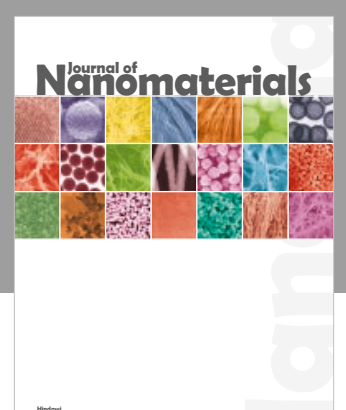

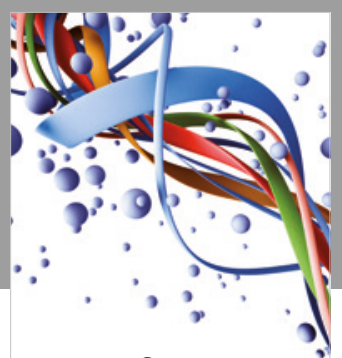

Scientifica

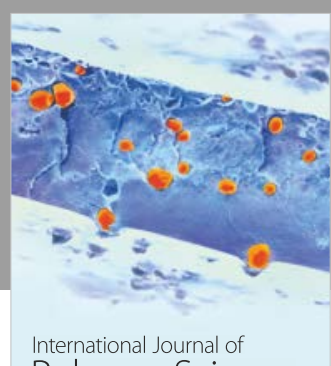

Polymer Science

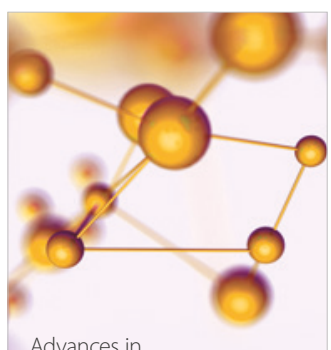

Physical Chemistry
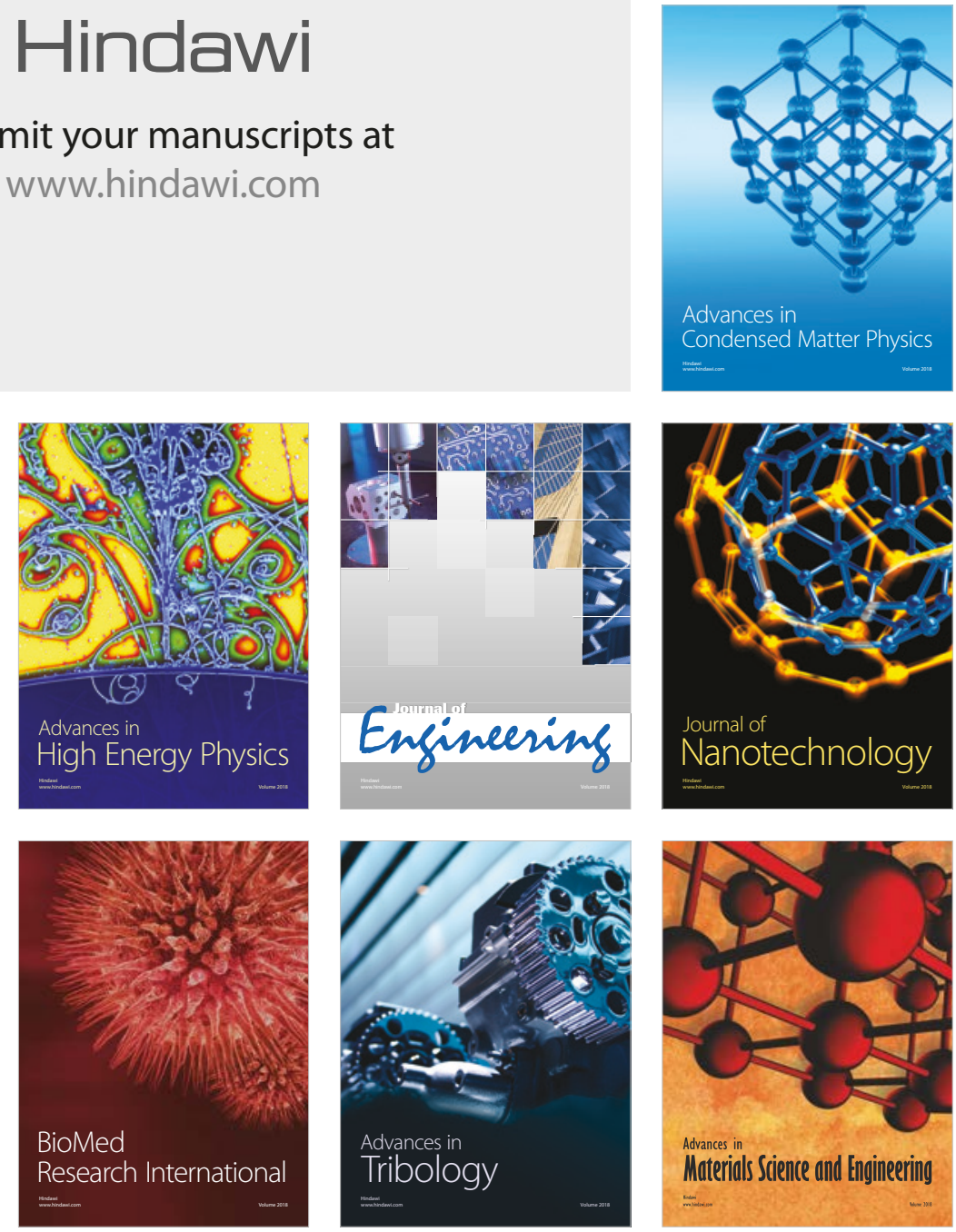